\title{
SCA Medium: A New Culture Medium for the Isolation of All Candida auris Clades
}

\author{
Ahmad Ibrahim ${ }^{1,2}$, Lucie Peyclit ${ }^{1,2}$, Rim Abdallah ${ }^{1,2}$, Saber Khelaifia ${ }^{1,2}{ }^{\circ}$, Amanda Chamieh ${ }^{1,2}(\mathbb{O}$, \\ Jean-Marc Rolain 1,2 and Fadi Bittar 1,2,*(D) \\ 1 Aix Marseille University, IRD, APHM, MEPHI, 13005 Marseille, France; \\ ahmad.ibrahim@etu.univ-amu.fr (A.I.); lucie.peyclit@ap-hm.fr (L.P.); rim.abdallah@etu.univ-amu.fr (R.A.); \\ saber.khelaifia@univ-amu.fr (S.K.); amanda.chamieh@etu.univ-amu.fr (A.C.); \\ jean-marc.rolain@univ-amu.fr (J.-M.R.) \\ 2 IHU-Méditerranée Infection, 13005 Marseille, France \\ * Correspondence: fadi.bittar@univ-amu.fr
}

Citation: Ibrahim, A.; Peyclit, L.; Abdallah, R.; Khelaifia, S.; Chamieh, A.; Rolain, J.-M.; Bittar, F. SCA Medium: A New Culture Medium for the Isolation of All Candida auris Clades. J. Fungi 2021, 7, 433. https:// doi.org/10.3390/jof7060433

Academic Editors: Jacques F. Meis and Anuradha Chowdhary

Received: 22 April 2021

Accepted: 28 May 2021

Published: 29 May 2021

Publisher's Note: MDPI stays neutral with regard to jurisdictional claims in published maps and institutional affiliations.

Copyright: () 2021 by the authors. Licensee MDPI, Basel, Switzerland. This article is an open access article distributed under the terms and conditions of the Creative Commons Attribution (CC BY) license (https:// creativecommons.org/licenses/by/ $4.0 /)$.

\begin{abstract}
Candida auris is an emerging multidrug-resistant yeast causing nosocomial infections and associated with high mortality in immunocompromised patients. Rapid identification and characterisation are necessary for diagnosis and containing its spread. In this study, we present a selective culture medium for all $C$. auris clades. This medium is sensitive with a limit of detection ranging between $10^{1}$ and $10^{2} \mathrm{CFU} / \mathrm{mL}$. The $100 \%$ specificity of SCA (specific C. auris) medium is confirmed on a set of 135 Candida strains, 50 bacterial species and 200 human stool samples. Thus, this medium specifically selects for $C$. auris isolation from clinical samples, allowing the latter to study its phenotypic profile.
\end{abstract}

Keywords: Candida; Candida auris; culture; emerging fungus; isolation; specific medium

\section{Introduction}

Candida auris is an emerging multidrug-resistant pathogen that was first isolated in 2009 [1] and is now known to have four geographical clades: South Asia: India; East Asia: Japan; Southern Africa: South Africa; and South America: Venezuela [2]. Recently, a potential fifth clade (Clade V) has been described in Iran [3]. C. auris is a biofilm-forming, halotolerant, thermo-resistant yeast $[1,4]$ that can grow at temperatures ranging between $30{ }^{\circ} \mathrm{C}$ and $42{ }^{\circ} \mathrm{C}$ and can tolerate up to $10 \%$ salinity [5]. This promotes the ability of $\mathrm{C}$. auris to colonize various medical equipment, plastic surfaces, and nosocomial environments [2-4], rendering C. auris an invasive, powerful pathogen. In addition, C. auris is highly resistant to different classes of antifungal agents such as azoles, amphotericin $B$ and echinocandins [6] and is associated with significant mortality, especially in immunocompromised patients with multiple comorbidities such as diabetes mellitus, renal failure, and cardiovascular disease [2,4]. Thus, its rapid identification and characterization are necessary to optimize clinical outcomes and to attempt to contain its nosocomial and worldwide spread.

The diagnosis of $C$. auris is challenging. The vast majority of commercial, readily available diagnostics, such as VITEK, API20C-AUX, Auxa-Color 2, BD Phoenixand MicroScan are misleading. C. auris is misidentified as other Candida species, namely Candida famata, Candida haemulonii, Candida duobushaemulonii, Candida sake, Candida lustaniae and Candida guilliermondii among others $[7,8]$. This often leads to delays in appropriate management and treatment. However, the use of Matrix-Assisted Laser Desorption/Ionization Time of Flight Mass Spectrometry (MALDI-TOF-MS) is promising [7,8], since the spectrum of C. auris is available in the reference database. In addition, the user can carefully select, "hand-pick", and isolate the corresponding identified colonies from other colonies growing on a plate. Moreover, the molecular identification and characterization of C. auris is well 
developed [9], and the use of some real-time PCR-targeting C. auris and sequencing of the ITS region is promising [9-12]. In 2017, a modified Sabouraud medium was suggested by Welsh et al. for a specific isolation of $C$. auris [5]. However, some Candida isolates were still misidentified $[7,8]$. Thus, our aim was to develop a specific medium only selective for all $C$. auris clades from clinical samples.

\section{Materials and Methods}

We collected 135 fungal strains (29 different species) [13-15] (Table 1) and cultured them on the modified Sabouraud broth suggested by Welsh et al. that contains $20 \mathrm{~g} / \mathrm{L}$ Mannitol as a carbon source (in order to inhibit C. glabrata growth) [5] in both liquid and solid-phase media. For the liquid phase, turbidity of the broth was measured by spectrophotometry at 24,48 , and $72 \mathrm{~h}$ of incubation at $40{ }^{\circ} \mathrm{C}$. This served to control the growth of each tested strain. For the solid phase, we added autoclaved bacterial agar $(15 \mathrm{~g} / \mathrm{L})$ and adjusted for a $\mathrm{pH}=7$. Each colony was streaked directly on the solid agar or the liquid inoculum.

Table 1. Strains and samples tested on the SCA medium.

\begin{tabular}{|c|c|c|c|c|c|c|}
\hline Type & Strain/Sample & $\mathrm{Nb}$ & Source & Origin & Identification & $\begin{array}{l}\text { qPCR } C \text {. auris } \\
\text { (Ibrahim et al., 2021) }\end{array}$ \\
\hline \multirow{15}{*}{$\begin{array}{l}\text { Gram-positive } \\
\text { bacteria }\end{array}$} & Bacillus cereus & 1 & Clinical & $\begin{array}{l}\text { Marseille, } \\
\text { France }\end{array}$ & $\begin{array}{l}\text { MALDI-TOF- } \\
\text { MS }\end{array}$ & Not tested \\
\hline & $\begin{array}{l}\text { Corynebacterium } \\
\text { amycolatum }\end{array}$ & 1 & Clinical & $\begin{array}{l}\text { Marseille, } \\
\text { France }\end{array}$ & $\begin{array}{l}\text { MALDI-TOF- } \\
\text { MS }\end{array}$ & Not tested \\
\hline & $\begin{array}{l}\text { Corynebacterium } \\
\text { jeikeium }\end{array}$ & 1 & Clinical & $\begin{array}{l}\text { Marseille, } \\
\text { France }\end{array}$ & $\begin{array}{l}\text { MALDI-TOF- } \\
\text { MS }\end{array}$ & Not tested \\
\hline & $\begin{array}{l}\text { Corynebacterium } \\
\text { propinquum }\end{array}$ & 1 & Clinical & $\begin{array}{l}\text { Marseille, } \\
\text { France }\end{array}$ & $\begin{array}{l}\text { MALDI-TOF- } \\
\text { MS }\end{array}$ & Not tested \\
\hline & $\begin{array}{l}\text { Corynebacterium } \\
\text { striatum }\end{array}$ & 1 & Clinical & $\begin{array}{l}\text { Marseille, } \\
\text { France }\end{array}$ & $\begin{array}{l}\text { MALDI-TOF- } \\
\text { MS }\end{array}$ & Not tested \\
\hline & Enterococcus faecalis & 1 & Clinical & $\begin{array}{l}\text { Marseille, } \\
\text { France }\end{array}$ & $\begin{array}{l}\text { MALDI-TOF- } \\
\text { MS }\end{array}$ & Not tested \\
\hline & Enterococcus faecium & 1 & Clinical & $\begin{array}{l}\text { Marseille, } \\
\text { France }\end{array}$ & $\begin{array}{l}\text { MALDI-TOF- } \\
\text { MS }\end{array}$ & Not tested \\
\hline & Micrococcus luteus & 1 & Clinical & $\begin{array}{l}\text { Marseille, } \\
\text { France }\end{array}$ & $\begin{array}{l}\text { MALDI-TOF- } \\
\text { MS }\end{array}$ & Not tested \\
\hline & $\begin{array}{l}\text { Staphylococcus } \\
\text { aureus }\end{array}$ & 1 & Clinical & $\begin{array}{l}\text { Marseille, } \\
\text { France }\end{array}$ & $\begin{array}{l}\text { MALDI-TOF- } \\
\text { MS }\end{array}$ & Not tested \\
\hline & $\begin{array}{l}\text { Staphylococcus } \\
\text { capitis }\end{array}$ & 1 & Clinical & $\begin{array}{l}\text { Marseille, } \\
\text { France }\end{array}$ & $\begin{array}{l}\text { MALDI-TOF- } \\
\text { MS }\end{array}$ & Not tested \\
\hline & $\begin{array}{l}\text { Staphylococcus } \\
\text { cohnii }\end{array}$ & 1 & Clinical & $\begin{array}{l}\text { Marseille, } \\
\text { France }\end{array}$ & $\begin{array}{l}\text { MALDI-TOF- } \\
\text { MS }\end{array}$ & Not tested \\
\hline & $\begin{array}{l}\text { Staphylococcus } \\
\text { epidermidis }\end{array}$ & 1 & Clinical & $\begin{array}{l}\text { Marseille, } \\
\text { France }\end{array}$ & $\begin{array}{l}\text { MALDI-TOF- } \\
\text { MS }\end{array}$ & Not tested \\
\hline & $\begin{array}{l}\text { Staphylococcus } \\
\text { haemolyticus }\end{array}$ & 1 & Clinical & $\begin{array}{l}\text { Marseille, } \\
\text { France }\end{array}$ & $\begin{array}{l}\text { MALDI-TOF- } \\
\text { MS }\end{array}$ & Not tested \\
\hline & $\begin{array}{l}\text { Staphylococcus } \\
\text { lugdunensis }\end{array}$ & 1 & Clinical & $\begin{array}{l}\text { Marseille, } \\
\text { France }\end{array}$ & $\begin{array}{l}\text { MALDI-TOF- } \\
\text { MS }\end{array}$ & Not tested \\
\hline & $\begin{array}{l}\text { Staphylococcus } \\
\text { pasteuri }\end{array}$ & 1 & Clinical & $\begin{array}{l}\text { Marseille, } \\
\text { France }\end{array}$ & $\begin{array}{l}\text { MALDI-TOF- } \\
\text { MS }\end{array}$ & Not tested \\
\hline
\end{tabular}


Table 1. Cont.

\begin{tabular}{|c|c|c|c|c|c|c|}
\hline Type & Strain/Sample & $\mathrm{Nb}$ & Source & Origin & Identification & $\begin{array}{l}\text { qPCR C. auris } \\
\text { (Ibrahim et al., 2021) }\end{array}$ \\
\hline & $\begin{array}{l}\text { Staphylococcus } \\
\text { saprophyticus }\end{array}$ & 1 & Clinical & $\begin{array}{l}\text { Marseille, } \\
\text { France }\end{array}$ & $\begin{array}{l}\text { MALDI-TOF- } \\
\text { MS }\end{array}$ & Not tested \\
\hline & $\begin{array}{l}\text { Staphylococcus } \\
\text { simulans }\end{array}$ & 1 & Clinical & $\begin{array}{l}\text { Marseille, } \\
\text { France }\end{array}$ & $\begin{array}{l}\text { MALDI-TOF- } \\
\text { MS }\end{array}$ & Not tested \\
\hline & $\begin{array}{l}\text { Staphylococcus } \\
\text { warneri }\end{array}$ & 1 & Clinical & $\begin{array}{l}\text { Marseille, } \\
\text { France }\end{array}$ & $\begin{array}{l}\text { MALDI-TOF- } \\
\text { MS }\end{array}$ & Not tested \\
\hline & $\begin{array}{l}\text { Streptococcus } \\
\text { agalactiae }\end{array}$ & 1 & Clinical & $\begin{array}{l}\text { Marseille, } \\
\text { France }\end{array}$ & $\begin{array}{l}\text { MALDI-TOF- } \\
\text { MS }\end{array}$ & Not tested \\
\hline & $\begin{array}{l}\text { Streptococcus } \\
\text { dysgalactiae }\end{array}$ & 1 & Clinical & $\begin{array}{l}\text { Marseille, } \\
\text { France }\end{array}$ & $\begin{array}{l}\text { MALDI-TOF- } \\
\text { MS }\end{array}$ & Not tested \\
\hline & $\begin{array}{l}\text { Streptococcus } \\
\text { equinus }\end{array}$ & 1 & Clinical & $\begin{array}{l}\text { Marseille, } \\
\text { France }\end{array}$ & $\begin{array}{l}\text { MALDI-TOF- } \\
\text { MS }\end{array}$ & Not tested \\
\hline & Streptococcus mitis & 1 & Clinical & $\begin{array}{l}\text { Marseille, } \\
\text { France }\end{array}$ & $\begin{array}{l}\text { MALDI-TOF- } \\
\text { MS }\end{array}$ & Not tested \\
\hline & $\begin{array}{l}\text { Streptococcus } \\
\text { pneumoniae }\end{array}$ & 1 & Clinical & $\begin{array}{l}\text { Marseille, } \\
\text { France }\end{array}$ & $\begin{array}{l}\text { MALDI-TOF- } \\
\text { MS }\end{array}$ & Not tested \\
\hline & $\begin{array}{l}\text { Staphylococcus } \\
\text { hominis }\end{array}$ & 1 & Clinical & $\begin{array}{l}\text { Marseille, } \\
\text { France }\end{array}$ & $\begin{array}{l}\text { MALDI-TOF- } \\
\text { MS }\end{array}$ & Not tested \\
\hline & $\begin{array}{l}\text { Streptococcus } \\
\text { salivarius }\end{array}$ & 1 & Clinical & $\begin{array}{l}\text { Marseille, } \\
\text { France }\end{array}$ & $\begin{array}{l}\text { MALDI-TOF- } \\
\text { MS }\end{array}$ & Not tested \\
\hline Subtotal & & 25 & & & & \\
\hline \multirow{16}{*}{$\begin{array}{l}\text { Gram-negative } \\
\text { bacteria }\end{array}$} & $\begin{array}{l}\text { Achromobacter } \\
\text { xylosoxidans }\end{array}$ & 1 & Clinical & $\begin{array}{l}\text { Marseille, } \\
\text { France }\end{array}$ & $\begin{array}{l}\text { MALDI-TOF- } \\
\text { MS }\end{array}$ & Not tested \\
\hline & $\begin{array}{l}\text { Acinetobacter } \\
\text { baumannii }\end{array}$ & 1 & Clinical & $\begin{array}{l}\text { Marseille, } \\
\text { France }\end{array}$ & $\begin{array}{l}\text { MALDI-TOF- } \\
\text { MS }\end{array}$ & Not tested \\
\hline & Bacteroides fragilis & 1 & Clinical & $\begin{array}{l}\text { Marseille, } \\
\text { France }\end{array}$ & $\begin{array}{l}\text { MALDI-TOF- } \\
\text { MS }\end{array}$ & Not tested \\
\hline & Citrobacter braakii & 1 & Clinical & $\begin{array}{l}\text { Marseille, } \\
\text { France }\end{array}$ & $\begin{array}{l}\text { MALDI-TOF- } \\
\text { MS }\end{array}$ & Not tested \\
\hline & Citrobacter freundii & 1 & Clinical & $\begin{array}{l}\text { Marseille, } \\
\text { France }\end{array}$ & $\begin{array}{l}\text { MALDI-TOF- } \\
\text { MS }\end{array}$ & Not tested \\
\hline & Citrobacter koseri & 1 & Clinical & $\begin{array}{l}\text { Marseille, } \\
\text { France }\end{array}$ & $\begin{array}{l}\text { MALDI-TOF- } \\
\text { MS }\end{array}$ & Not tested \\
\hline & $\begin{array}{l}\text { Enterobacter } \\
\text { aerogenes }\end{array}$ & 1 & Clinical & $\begin{array}{l}\text { Marseille, } \\
\text { France }\end{array}$ & $\begin{array}{l}\text { MALDI-TOF- } \\
\text { MS }\end{array}$ & Not tested \\
\hline & Enterobacter asburiae & 1 & Clinical & $\begin{array}{l}\text { Marseille, } \\
\text { France }\end{array}$ & $\begin{array}{l}\text { MALDI-TOF- } \\
\text { MS }\end{array}$ & Not tested \\
\hline & Enterobacter cloacae & 1 & Clinical & $\begin{array}{l}\text { Marseille, } \\
\text { France }\end{array}$ & $\begin{array}{l}\text { MALDI-TOF- } \\
\text { MS }\end{array}$ & Not tested \\
\hline & Enterobacter kobeii & 1 & Clinical & $\begin{array}{l}\text { Marseille, } \\
\text { France }\end{array}$ & $\begin{array}{l}\text { MALDI-TOF- } \\
\text { MS }\end{array}$ & Not tested \\
\hline & Escherichia coli & 1 & Clinical & $\begin{array}{l}\text { Marseille, } \\
\text { France }\end{array}$ & $\begin{array}{l}\text { MALDI-TOF- } \\
\text { MS }\end{array}$ & Not tested \\
\hline & $\begin{array}{l}\text { Haemophilus } \\
\text { influenzae }\end{array}$ & 1 & Clinical & $\begin{array}{l}\text { Marseille, } \\
\text { France }\end{array}$ & $\begin{array}{l}\text { MALDI-TOF- } \\
\text { MS }\end{array}$ & Not tested \\
\hline & $\begin{array}{l}\text { Haemophilus } \\
\text { parainfluenzae }\end{array}$ & 1 & Clinical & $\begin{array}{l}\text { Marseille, } \\
\text { France }\end{array}$ & $\begin{array}{l}\text { MALDI-TOF- } \\
\text { MS }\end{array}$ & Not tested \\
\hline & Hafnia alvei & 1 & Clinical & $\begin{array}{l}\text { Marseille, } \\
\text { France }\end{array}$ & $\begin{array}{l}\text { MALDI-TOF- } \\
\text { MS }\end{array}$ & Not tested \\
\hline & Klebsiella oxytoca & 1 & Clinical & $\begin{array}{l}\text { Marseille, } \\
\text { France }\end{array}$ & $\begin{array}{l}\text { MALDI-TOF- } \\
\text { MS }\end{array}$ & Not tested \\
\hline & $\begin{array}{l}\text { Klebsiella } \\
\text { pneumoniae }\end{array}$ & 1 & Clinical & $\begin{array}{l}\text { Marseille, } \\
\text { France }\end{array}$ & $\begin{array}{l}\text { MALDI-TOF- } \\
\text { MS }\end{array}$ & Not tested \\
\hline
\end{tabular}


Table 1. Cont.

\begin{tabular}{|c|c|c|c|c|c|c|}
\hline Type & Strain/Sample & $\mathrm{Nb}$ & Source & Origin & Identification & $\begin{array}{l}\text { qPCR } C \text {. auris } \\
\text { (Ibrahim et al., 2021) }\end{array}$ \\
\hline & Moraxella catarrhalis & 1 & Clinical & $\begin{array}{l}\text { Marseille, } \\
\text { France }\end{array}$ & $\begin{array}{l}\text { MALDI-TOF- } \\
\text { MS }\end{array}$ & Not tested \\
\hline & Morganella morganii & 1 & Clinical & $\begin{array}{l}\text { Marseille, } \\
\text { France }\end{array}$ & $\begin{array}{l}\text { MALDI-TOF- } \\
\text { MS }\end{array}$ & Not tested \\
\hline & Pasteurella multocida & 1 & Clinical & $\begin{array}{l}\text { Marseille, } \\
\text { France }\end{array}$ & $\begin{array}{l}\text { MALDI-TOF- } \\
\text { MS }\end{array}$ & Not tested \\
\hline & Proteus mirabilis & 1 & Clinical & $\begin{array}{l}\text { Marseille, } \\
\text { France }\end{array}$ & $\begin{array}{l}\text { MALDI-TOF- } \\
\text { MS }\end{array}$ & Not tested \\
\hline & Proteus vulgaris & 1 & Clinical & $\begin{array}{l}\text { Marseille, } \\
\text { France }\end{array}$ & $\begin{array}{l}\text { MALDI-TOF- } \\
\text { MS }\end{array}$ & Not tested \\
\hline & Providencia stuartii & 1 & Clinical & $\begin{array}{l}\text { Marseille, } \\
\text { France }\end{array}$ & $\begin{array}{l}\text { MALDI-TOF- } \\
\text { MS }\end{array}$ & Not tested \\
\hline & $\begin{array}{l}\text { Pseudomonas } \\
\text { aeruginosa }\end{array}$ & 1 & Clinical & $\begin{array}{l}\text { Marseille, } \\
\text { France }\end{array}$ & $\begin{array}{l}\text { MALDI-TOF- } \\
\text { MS }\end{array}$ & Not tested \\
\hline & $\begin{array}{l}\text { Raoultella } \\
\text { ornithinolytica }\end{array}$ & 1 & Clinical & $\begin{array}{l}\text { Marseille, } \\
\text { France }\end{array}$ & $\begin{array}{l}\text { MALDI-TOF- } \\
\text { MS }\end{array}$ & Not tested \\
\hline & $\begin{array}{l}\text { Stenotrophomonas } \\
\text { maltophilis }\end{array}$ & 1 & Clinical & $\begin{array}{l}\text { Marseille, } \\
\text { France }\end{array}$ & $\begin{array}{l}\text { MALDI-TOF- } \\
\text { MS }\end{array}$ & Not tested \\
\hline Subtotal & & 25 & & & & \\
\hline \multirow{16}{*}{ Yeast } & Candida albicans & 73 & Clinical & $\begin{array}{l}\text { Marseille, } \\
\text { France }\end{array}$ & $\begin{array}{l}\text { MALDI-TOF- } \\
\text { MS }\end{array}$ & Not tested \\
\hline & Candida glabrata & 8 & Clinical & $\begin{array}{l}\text { Marseille, } \\
\text { France }\end{array}$ & $\begin{array}{l}\text { MALDI-TOF- } \\
\text { MS }\end{array}$ & Not tested \\
\hline & Candida krusei & 4 & Clinical & $\begin{array}{l}\text { Marseille, } \\
\text { France }\end{array}$ & $\begin{array}{l}\text { MALDI-TOF- } \\
\text { MS }\end{array}$ & Not tested \\
\hline & Candida parapsilosis & 6 & Clinical & $\begin{array}{l}\text { Marseille, } \\
\text { France }\end{array}$ & $\begin{array}{l}\text { MALDI-TOF- } \\
\text { MS }\end{array}$ & Not tested \\
\hline & Candida lusitaniae & 3 & Clinical & $\begin{array}{l}\text { Marseille, } \\
\text { France }\end{array}$ & $\begin{array}{l}\text { MALDI-TOF- } \\
\text { MS }\end{array}$ & Not tested \\
\hline & Candida tropicalis & 6 & Clinical & $\begin{array}{l}\text { Marseille, } \\
\text { France }\end{array}$ & $\begin{array}{l}\text { MALDI-TOF- } \\
\text { MS }\end{array}$ & Not tested \\
\hline & Candida zelanoides & 1 & Clinical & $\begin{array}{l}\text { Marseille, } \\
\text { France }\end{array}$ & $\begin{array}{l}\text { MALDI-TOF- } \\
\text { MS }\end{array}$ & Not tested \\
\hline & Candida lipolytica & 1 & Clinical & $\begin{array}{l}\text { Marseille, } \\
\text { France }\end{array}$ & $\begin{array}{l}\text { MALDI-TOF- } \\
\text { MS }\end{array}$ & Not tested \\
\hline & Candida inconspicua & 1 & Clinical & $\begin{array}{l}\text { Marseille, } \\
\text { France }\end{array}$ & $\begin{array}{l}\text { MALDI-TOF- } \\
\text { MS }\end{array}$ & Not tested \\
\hline & Candida intermedia & 1 & Clinical & $\begin{array}{l}\text { Marseille, } \\
\text { France }\end{array}$ & $\begin{array}{l}\text { MALDI-TOF- } \\
\text { MS }\end{array}$ & Not tested \\
\hline & $\begin{array}{l}\text { Candida } \\
\text { guilliermondii }\end{array}$ & 3 & Clinical & $\begin{array}{l}\text { Marseille, } \\
\text { France }\end{array}$ & $\begin{array}{l}\text { MALDI-TOF- } \\
\text { MS }\end{array}$ & Not tested \\
\hline & Candida bracarensis & 1 & Clinical & $\begin{array}{l}\text { Marseille, } \\
\text { France }\end{array}$ & $\begin{array}{l}\text { MALDI-TOF- } \\
\text { MS }\end{array}$ & Not tested \\
\hline & Candida utilis & 1 & Clinical & $\begin{array}{l}\text { Marseille, } \\
\text { France }\end{array}$ & $\begin{array}{l}\text { MALDI-TOF- } \\
\text { MS }\end{array}$ & Not tested \\
\hline & Candida bovina & 1 & Clinical & $\begin{array}{l}\text { Marseille, } \\
\text { France }\end{array}$ & $\begin{array}{l}\text { MALDI-TOF- } \\
\text { MS }\end{array}$ & Not tested \\
\hline & Candida dubliniensis & 2 & Clinical & $\begin{array}{l}\text { Marseille, } \\
\text { France }\end{array}$ & $\begin{array}{l}\text { MALDI-TOF- } \\
\text { MS }\end{array}$ & Not tested \\
\hline & Candida norvegensis & 1 & Clinical & $\begin{array}{l}\text { Marseille, } \\
\text { France }\end{array}$ & $\begin{array}{l}\text { MALDI-TOF- } \\
\text { MS }\end{array}$ & Not tested \\
\hline
\end{tabular}


Table 1. Cont.

\begin{tabular}{|c|c|c|c|c|c|c|}
\hline Type & Strain/Sample & $\mathrm{Nb}$ & Source & Origin & Identification & $\begin{array}{l}\text { qPCR } C \text {. auris } \\
\text { (Ibrahim et al., 2021) }\end{array}$ \\
\hline & Candida kefyr & 2 & Clinical & $\begin{array}{l}\text { Marseille, } \\
\text { France }\end{array}$ & $\begin{array}{l}\text { MALDI-TOF- } \\
\text { MS }\end{array}$ & Not tested \\
\hline & Candida beverwijkiae & 1 & Clinical & $\begin{array}{l}\text { Marseille, } \\
\text { France }\end{array}$ & $\begin{array}{l}\text { MALDI-TOF- } \\
\text { MS }\end{array}$ & Not tested \\
\hline & $\begin{array}{l}\text { Cryptococcus } \\
\text { diffluens }\end{array}$ & 1 & Clinical & $\begin{array}{l}\text { Marseille, } \\
\text { France }\end{array}$ & $\begin{array}{l}\text { MALDI-TOF- } \\
\text { MS }\end{array}$ & Not tested \\
\hline & $\begin{array}{l}\text { Cryptococcus } \\
\text { uniguttulatus }\end{array}$ & 1 & Clinical & $\begin{array}{l}\text { Marseille, } \\
\text { France }\end{array}$ & $\begin{array}{l}\text { MALDI-TOF- } \\
\text { MS }\end{array}$ & Not tested \\
\hline & $\begin{array}{l}\text { Cryptococcus } \\
\text { neoformans }\end{array}$ & 2 & Clinical & $\begin{array}{l}\text { Marseille, } \\
\text { France }\end{array}$ & $\begin{array}{l}\text { MALDI-TOF- } \\
\text { MS }\end{array}$ & Not tested \\
\hline & $\begin{array}{l}\text { Saccharomyces } \\
\text { cerevisiae }\end{array}$ & 2 & Clinical & $\begin{array}{l}\text { Marseille, } \\
\text { France }\end{array}$ & $\begin{array}{l}\text { MALDI-TOF- } \\
\text { MS }\end{array}$ & Not tested \\
\hline & $\begin{array}{l}\text { Rhodotorula } \\
\text { mucilaginosa }\end{array}$ & 1 & Clinical & $\begin{array}{l}\text { Marseille, } \\
\text { France }\end{array}$ & $\begin{array}{l}\text { MALDI-TOF- } \\
\text { MS }\end{array}$ & Not tested \\
\hline & Yarrowia lipolitica & 1 & Clinical & $\begin{array}{l}\text { Marseille, } \\
\text { France }\end{array}$ & $\begin{array}{l}\text { MALDI-TOF- } \\
\text { MS }\end{array}$ & Not tested \\
\hline & Candida haemulonii & 1 & Clinical & Netherlands & $\begin{array}{l}\text { MALDI-TOF- } \\
\text { MS }\end{array}$ & Not tested \\
\hline & $\begin{array}{l}\text { Candida } \\
\text { duobushaemulonii }\end{array}$ & 1 & Clinical & Netherlands & $\begin{array}{l}\text { MALDI-TOF- } \\
\text { MS }\end{array}$ & Not tested \\
\hline & Trichosporon asahii & 1 & Clinical & $\begin{array}{l}\text { Marseille, } \\
\text { France }\end{array}$ & $\begin{array}{l}\text { MALDI-TOF- } \\
\text { MS }\end{array}$ & Not tested \\
\hline & Kodamaea ohmeri & 1 & Clinical & $\begin{array}{l}\text { Marseille, } \\
\text { France }\end{array}$ & $\begin{array}{l}\text { MALDI-TOF- } \\
\text { MS }\end{array}$ & Not tested \\
\hline & $\begin{array}{l}\text { Candida auris Clade } \\
\text { I }\end{array}$ & 2 & Clinical & Netherlands & $\begin{array}{l}\text { MALDI-TOF- } \\
\text { MS }\end{array}$ & Positive \\
\hline & $\begin{array}{l}\text { Candida auris Clade } \\
\text { II }\end{array}$ & 1 & Clinical & $\begin{array}{l}\text { DSMZ } \\
\text { collection }\end{array}$ & $\begin{array}{l}\text { MALDI-TOF- } \\
\text { MS }\end{array}$ & Positive \\
\hline & $\begin{array}{l}\text { Candida auris Clade } \\
\text { III }\end{array}$ & 2 & Clinical & Netherlands & $\begin{array}{l}\text { MALDI-TOF- } \\
\text { MS }\end{array}$ & Positive \\
\hline & $\begin{array}{l}\text { Candida auris Clade } \\
\text { IV }\end{array}$ & 2 & Clinical & Netherlands & $\begin{array}{l}\text { MALDI-TOF- } \\
\text { MS }\end{array}$ & Positive \\
\hline Subtotal & & 135 & & & & \\
\hline $\begin{array}{l}\text { Human } \\
\text { samples }\end{array}$ & Stool samples & 200 & Clinical & $\begin{array}{l}\text { Marseille, } \\
\text { France }\end{array}$ & & Negative \\
\hline Total & & 385 & & & & \\
\hline
\end{tabular}

We then cultured all C. auris $(n=7)$ and Candida tropicalis $(n=6)$ (see results) strains on the different culture media used in our diagnostic laboratories. The media used for culturing yeasts were: Sabouraud Dextrose Agar, CHROMagar Candida and Buffered Charcoal Yeast Extract. The media used for culturing bacteria were: Chocolate agar, Tryptic Soy agar, Columbia agar, Mannitol Salt agar, MacConkey agar (BioMérieux, Marcy-l'Etoile, France) and LBJMR (Lucie Bardet and Jean-Marc Rolain) medium [16].

Finally, we cultured this set of 13 strains (i.e., C. tropicalis and C. auris) on different combinations of the Welsh et al. broth and MacConkey (MCK) agar, a selective medium for Gram-negative bacteria (see results and discussion) (Table 2) [17]. We prepared several media with varying concentrations of bile salts $(1.5 \mathrm{~g} / \mathrm{L}, 1 \mathrm{~g} / \mathrm{L}, 0.75 \mathrm{~g} / \mathrm{L})$ and / or crystal violet $(0.5 \mathrm{mg} / \mathrm{L}$ ) (used as inhibitors in MCK) [17] to the initial broth composition of Welsh et al. in solid and liquid phase with shaking (300 rpm) at $40{ }^{\circ} \mathrm{C}$ (Table 2). 
Table 2. Growth results of $C$. auris (seven strains from four clades) and $C$. tropicalis $(n=6)$ tested strains according to crystal violet and bile salts concentrations for each condition $5 \mathrm{~g}$ of pancreatic digest of casein. $5 \mathrm{~g}$ of peptic digest of animal tissue, $100 \mathrm{~g} \mathrm{NaCl}, 20 \mathrm{~g}$ of Mannitol, $50 \mathrm{mg} / \mathrm{L}$ chloramphenicol and $50 \mathrm{mg} / \mathrm{L}$ gentamicin were added.

\begin{tabular}{ccccccc}
\hline $\begin{array}{c}\text { Crystal } \\
\text { Violet }\end{array}$ & Bile Salts & $\begin{array}{c}\text { C.auris } \\
\text { Clade I }\end{array}$ & $\begin{array}{c}\text { C.auris } \\
\text { Clade II }\end{array}$ & $\begin{array}{c}\text { C.auris } \\
\text { Clade III }\end{array}$ & $\begin{array}{c}\text { C.auris } \\
\text { Clade VI }\end{array}$ & $\begin{array}{c}\text { C. } \\
\text { tropicalis }\end{array}$ \\
\hline $0 \mathrm{mg} / \mathrm{L}$ & $0 \mathrm{~g} / \mathrm{L}$ & ++ & ++ & ++ & ++ & ++ \\
$0 \mathrm{mg} / \mathrm{L}$ & $0.5 \mathrm{~g} / \mathrm{L}$ & ++ & ++ & ++ & ++ & ++ \\
$0 \mathrm{mg} / \mathrm{L}$ & $1 \mathrm{~g} / \mathrm{L}$ & ++ & ++ & ++ & ++ & ++ \\
$0 \mathrm{mg} / \mathrm{L}$ & $1.5 \mathrm{~g} / \mathrm{L}$ & ++ & + & ++ & ++ & + \\
$0.5 \mathrm{mg} / \mathrm{L}$ & $0 \mathrm{~g} / \mathrm{L}$ & ++ & ++ & ++ & ++ & - \\
$0.5 \mathrm{mg} / \mathrm{L}$ & $0.5 \mathrm{~g} / \mathrm{L}$ & ++ & ++ & ++ & ++ & - \\
$0.5 \mathrm{mg} / \mathrm{L}$ & $1 \mathrm{~g} / \mathrm{L}$ & ++ & + & ++ & ++ & - \\
$0.5 \mathrm{mg} / \mathrm{L}$ & $1.5 \mathrm{~g} / \mathrm{L}$ & ++ & + & ++ & ++ & - \\
\hline
\end{tabular}

To study the specificity of our designated medium, we cultured for 3 days at $40{ }^{\circ} \mathrm{C}$ a panel of various Gram-positive and Gram-negative bacteria $(n=50)$, different $C$. auris clades (DSM 21092 and 6 strains that were kindly provided by Dr. Jacques F Meis, Canisius Wilhemina Hospital, Department of Medical Microbiology and Infectious Diseases, the Netherlands) [9,18-20]. All C. auris strains were isolated from blood cultures except JCM 15,448 (Clade II), which is from the external ear canal. We tested also other Candida strains (including the closest Candida species to C. auris (C. haemulonii and C. duobushaemulonii) (directly streaked on the agar) [2,4]. In addition, we tested 200 fecal samples that were negative by real-time PCR for $C$. auris. These samples were collected at the Marseille Hospital (AP-HM, Assistance Publique-Hôpitaux Marseille) from routine laboratory diagnostics (Table 1). Given that this work did not involve human body/tissues or use clinical data from patients, and according to French law (Loi no 2012-300 of 5 March 2012 and Décret no 2016-1537 of 16 November 2016 published in the 'Journal Officiel de la République Française'), neither institutional ethical approval nor individual patient consent was required for this non-invasive study.

All patients routinely sign an approval that the samples they submit for testing at AP-HM labs may be used in research. Each stool sample was enriched in Tryptic soy broth (TSB) for 3 days at $37^{\circ} \mathrm{C}$. Then, a total of $0.1 \mathrm{~mL}$ of each enriched media was streaked on the solid media. All bacterial and fungal strains used in this work were selected from previous studies and identified correctly by MALDI-TOF-MS [13-15,21].

Moreover, to determine the sensitivity of our $C$. auris-specific medium, a series of ten-fold dilution $\left(10^{-1}\right.$ to $\left.10^{-10}\right)$ of $0.5 \mathrm{McFarland}$ of each $C$. auris clade was cultured. For the second clade, we tested the strain of DSMZ collection: DSM 21092. In addition, we also cultured a mixture of the same ten-fold dilutions of $0.5 \mathrm{McFarland}$ of each $C$. auris strain with a stool sample negative in real-time PCR for C. auris $\left(10^{-1}\right.$ to $\left.10^{-10}\right)$. We did this as an attempt to determine whether the natural presence of microbes in stools would affect the growth of $C$. auris on our designated medium.

\section{Results}

We first cultured all our available Candida spp. strains $(n=135)$ on the broth developed by Welsh et al. Interestingly, we isolated $100 \%$ of the tested C. auris $(n=7)$ and $C$. tropicalis $(n=6)$ species on the Welsh broth [5].

However, when we cultured all strains on a combination of MacConkey agar and the Welsh broth, the growth of $C$. tropicalis strains was totally inhibited. On the other hand, we observed a normal growth of all C. auris species.

The addition of crystal violet, with or without bile salts, did not affect the growth of C. auris (Figure 1), which we evaluated by measuring the turbidity of the broth using a spectrophotometer. However, we observed a total inhibition of all C. tropicalis strains in the presence of $0.5 \mathrm{mg} / \mathrm{L}$ crystal violet. 


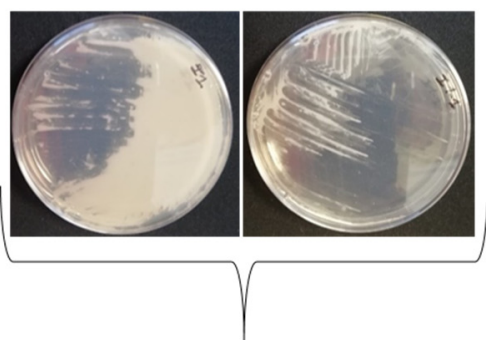

Clade I : India

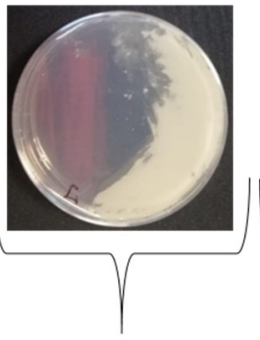

Clade II : Japan

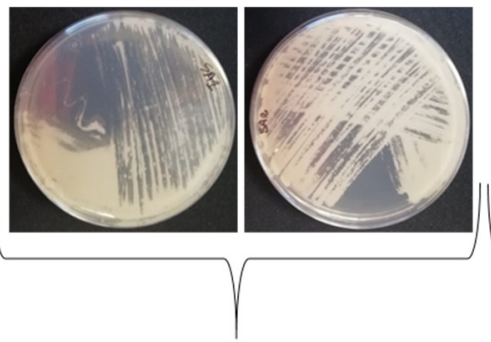

Clade III : South africa

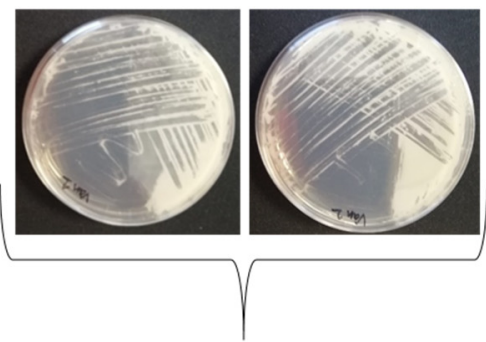

Clade IV : Venezuela

Figure 1. Striking Candida auris strains onto SCA medium, 2 strains/clade except for C. auris clade II (one strain).

The addition of bile salts only partially inhibited the growth of $C$. auris clade II. This was reflected as a decrease in the spectrophotometer value in comparison to the negative control only containing broth and crystal violet. As for $C$. tropicalis strains, the presence of bile salts without crystal violet did not inhibit their growth. It was then excluded from the final composition of the SCA medium (Table 2).

Therefore, the growth of $C$. tropicalis was inhibited by adding crystal violet at $0.5 \mathrm{mg} / \mathrm{L}$ to the initial broth suggested by Welsh et al. with no effect on the growth of the $7 \mathrm{C}$. auris strains (2 strains/clade: except for clade II (one strain)) in solid and liquid phase.

Moreover, the specificity of this medium was also confirmed after cultivating all bacterial spp. Candida spp. and fecal samples mentioned in Table 1 on the last composition of our medium (Table 3). No growth was noted after 3 days of incubation at $40^{\circ} \mathrm{C}$.

Table 3. Final composition of SCA (Specific Candida auris) medium.

\begin{tabular}{ccccccccc}
\hline & $\begin{array}{c}\text { Pancreatic } \\
\text { Digest of } \\
\text { Casein }\end{array}$ & $\begin{array}{c}\text { Peptic Digest of } \\
\text { Animal Tissue }\end{array}$ & NaCl & Mannitol & $\begin{array}{c}\text { Crystal } \\
\text { Violet }\end{array}$ & Agar & pH & Chloramphenicol \\
\hline $\begin{array}{c}\text { Welsh et al. } \\
\text { broth }\end{array}$ & $5 \mathrm{~g}$ & $5 \mathrm{~g}$ & $100 \mathrm{~g}$ & $20 \mathrm{~g}$ & - & 5.6 & $50 \mathrm{mg} / \mathrm{L}$ \\
$\begin{array}{c}\text { SCA } \\
\text { medium }\end{array}$ & $5 \mathrm{~g}$ & $5 \mathrm{~g}$ & $100 \mathrm{~g}$ & $20 \mathrm{~g}$ & $0.5 \mathrm{mg}$ & $15 \mathrm{~g}$ & 7 & $50 \mathrm{mg} / \mathrm{L}$ \\
\hline
\end{tabular}

Concerning the sensitivity of our medium, after cultivation of several dilutions of a solution of $0.5 \mathrm{McF}$ arland $\left(10^{-1}\right.$ to $\left.10^{-10}\right)$, the limit of detection (LOD) of $C$. auris Clade I, II and VI was $10^{2} \mathrm{CFU} / \mathrm{mL}$ in both serial dilutions (with physiological water and a fecal sample). For the third clade (South Africa), we observed an excessive growth of both strains at an LOD of $10^{1} \mathrm{CFU} / \mathrm{mL}$. Thus, the presence of other microbes in a fecal sample does not inhibit or affect the growth of $C$. auris. All resulting colonies were isolated and subsequently identified by MALDI-TOF-MS and real-time PCR [9].

The final composition of our medium in $1 \mathrm{~L}$ of deionized water was $5 \mathrm{~g}$ of pancreatic digest of casein, $5 \mathrm{~g}$ of peptic digest of animal tissue, $20 \mathrm{~g}$ of mannitol, $0.5 \mathrm{mg}$ of crystal violet (Sigma-aldrich, Darmstadt, Germany), $100 \mathrm{~g} \mathrm{NaCl}, 50 \mathrm{mg} / \mathrm{L}$ of chloramphenicol and $50 \mathrm{mg} / \mathrm{L}$ of gentamicin with $\mathrm{pH}=7( \pm 0.2)$ at $40{ }^{\circ} \mathrm{C}$ (Figure 1 , Table 3 ).

\section{Discussion}

Correct identification and rapid isolation of $C$. auris is essential for the timely and appropriate antifungal treatment and for infection prevention and control measures. This ensures limiting and controlling a possible nosocomial outbreak of a pathogen with fatal consequences and limited therapeutic options. This need is emphasized in the current COVID-19 pandemic we are living in, whereby an increasing amount of critically ill, immunocompromised patients populate the hospitals [22-24]. The gold standard of diagnosis remains molecular identification by sequencing and/or real-time PCR [9-12]. However, this is expensive and may only be available at specialized referral or research laboratories. Therefore, we have developed a cheap, simple, easily-prepared medium for isolating $C$. auris that is specific and sensitive. 
A caveat to the enrichment broth developed by Welsh et al. for C. auris isolation is that it also grows $C$. tropicalis strains, which we demonstrated in our work. We show that the addition of crystal violet $(0.5 \mathrm{mg} / \mathrm{L})$ to our specific $C$. auris medium inhibited the growth of C. tropicalis. SCA (specific C. auris) is considered as a new version of the above-mentioned broth [5].

In addition, other selective media are recently described for the isolation of C. auris [25-27]. Generally, the selected criteria to cultivate this yeast remain the same as those used in our work: the thermo-resistance, halo-tolerance and multi-resistance to many antifungal agents [2,4]. Here, we managed to develop a medium with the minimum possible inhibitors $\left(40{ }^{\circ} \mathrm{C}\right.$ incubation instead of 42 , and $10 \%$ salinity instead of $12.5 \%$ compared to another study [26]). These moderate inhibitors still maintain the selectivity of SCA medium for all C. auris.

Usually, C. auris, C. krusei and C. parapsilosis appear as pink colonies on the CHROMagar medium $[2,4,28]$, and a newer version of CHROMagar has been developed to identify $C$. auris $[25,27]$. This phenotypic identification may be biased and reader-dependent, since it is based on interpretation of the color/morphology of growing colonies. Moreover, a longer duration of incubation may lead to a change in results as well. Thus, a high percentage of error may occur. Our SCA medium facilitates the interpretation of results by only selecting for C. auris.

Interestingly, spiking a stool sample with a C. auris strain affected neither the specificity nor the sensitivity of this medium. However, increasing our sample size and testing a larger amount of clinical C. auris isolates and related yeasts (such as C. famata, C. pseudohaemulonii, C. metapsilosis, C. orthopsilosis, C. rugosa, C. vulturna, Wickerhamomyces anomalus and C. sake) is necessary for further evaluation, validation and reproducibility of our C. aurisspecific medium.

\section{Conclusions}

The use of our developed medium enables the rapid, specific isolation of C. auris strains and helps in the timely management of patients and resources to limit the occurrence of C. auris outbreaks. We propose an implementation of SCA medium in routine clinical mycology for screening skin, urine, vaginal and blood samples, especially in high-risk populations $[13,15]$. This SCA medium will further enhance our understanding of the phenotypic characteristics of $C$. auris and future isolates, most importantly allowing an accurate study of their antifungal resistance profiles.

Author Contributions: Conceptualization, A.I., L.P., J.-M.R. and F.B.; methodology, A.I., R.A., S.K. and A.C.; writing—original draft preparation, A.I., L.P. and F.B.; writing—review and editing, J.-M.R. and F.B.; supervision, J.-M.R. and F.B. All authors have read and agreed to the published version of the manuscript.

Funding: This work was supported by the French Government under the Investissements d'avenir (Investments for the Future) program managed by the Agence Nationale de la Recherche (ANR, fr: National Agency for Research), (reference: Méditerranée Infection 10-IAHU-03). This work was supported by Région Provence-Alpes-Côte d'Azur and European funding (FEDER (Fonds européen de développement régional) PRIMMI (Plateformes de Rechercheet d'Innovation Mutualisées Méditerranée Infection)).

Institutional Review Board Statement: Ethical review and approval were waived for this study, as this research does not involve human body/tissue, and only discarded stool samples, once laboratory analyses had been initiated, were used. According to French law (Loi no 2012-300 du 5 March 2012 and Décret no 2016-1537 du 16 November 2016 published in the 'Journal Officiel de la République Française'), no Institutional Review Board is necessary in this case.

Informed Consent Statement: Patient consent is not required for this type of study (Loi no 2012-300 of 5 March 2012 and Décret no 2016-1537 of 16 November 2016 published in the 'Journal Officiel de la République Française').

Data Availability Statement: Not applicable. 
Acknowledgments: We want to thank CookieTrad for English correction. We would like to thank Jacques F. Meis for sending us the strains of C. auris, C. haemulonii and C. duobushaemulonii.

Conflicts of Interest: The authors declare no conflict of interest.

\section{References}

1. Satoh, K.; Makimura, K.; Hasumi, Y.; Nishiyama, Y.; Uchida, K.; Yamaguchi, H. Candida auris sp. nov., a novel ascomycetous yeast isolated from the external ear canal of an inpatient in a Japanese hospital. Microbiol. Immunol. 2009, 53, 41-44. [CrossRef]

2. Osei Sekyere, J. Candida auris: A systematic review and meta-analysis of current updates on an emerging multidrug-resistant pathogen. Microbiologyopen 2018, 7, e00578. [CrossRef]

3. Chow, N.A.; De Groot, T.; Badali, H.; Abastabar, M.; Chiller, T.M.; Meis, J.F. Potential fifth clade of Candida auris, Iran, 2018. Emerg. Infect. Dis. 2019, 25, 1780-1781. [CrossRef]

4. Jeffery-Smith, A.; Taori, S.K.; Schelenz, S.; Jeffery, K.; Johnson, E.M.; Borman, A.; Manuel, R.; Browna, C.S. Candida auris: A review of the literature. Clin. Microbiol. Rev. 2018, 31, e00029-17. [CrossRef] [PubMed]

5. Welsh, R.M.; Bentz, M.L.; Shams, A.; Houston, H.; Lyons, A.; Rose, L.J.; Litvintseva, A.P. Survival, persistence, and isolation of the emerging multidrug-resistant pathogenic yeast Candida auris on a plastic health care surface. J. Clin. Microbiol. 2017, 55, 2996-3005. [CrossRef] [PubMed]

6. Rudramurthy, S.M.; Chakrabarti, A.; Paul, R.A.; Sood, P.; Kaur, H.; Capoor, M.R.; Kindo, A.J.; Marak, R.S.K.; Arora, A.; Sardana, R.; et al. Candida auris candidaemia in Indian ICUs: Analysis of risk factors. J. Antimicrob. Chemother. 2017, 72, 1794-1801. [CrossRef] [PubMed]

7. Kathuria, S.; Singh, P.K.; Sharma, C.; Prakash, A.; Masih, A.; Kumar, A.; Meis, J.F.; Chowdhary, A. Multidrug-resistant Candida auris misidentified as Candida haemulonii: Characterization by matrix-assisted laser desorption ionization-time of flight mass spectrometry and DNA sequencing and its antifungal susceptibility profile variability by vitek 2, CLSI broth microdilution, and etest method. J. Clin. Microbiol. 2015, 53, 1823-1830. [CrossRef] [PubMed]

8. Mizusawa, M.; Miller, H.; Green, R.; Lee, R.; Durante, M.; Perkins, R.; Hewitt, C.; Simner, P.J.; Carroll, K.C.; Hayden, R.T.; et al. Can multidrug-resistant candida auris be reliably identified in clinical microbiology laboratories? J. Clin. Microbiol. 2017, 55, 638-640. [CrossRef] [PubMed]

9. Ibrahim, A.; Baron, S.A.; Yousfi, H.; Hadjadj, L.; Lalaoui, R.; Morand, S.; Rolain, J.M.; Bittar, F. Development and standardization of a specific real-time PCR assay for the rapid detection of Candida auris. Eur. J. Clin. Microbiol. Infect. Dis. 2021, 1-5. [CrossRef]

10. Lima, A.; Widen, R.; Vestal, G.; Uy, D.; Silbert, S. A TaqMan probe-based real-time PCR assay for the rapid identification of the emerging multidrug-resistant pathogen candida auris on the BD max system. J. Clin. Microbiol. 2019, 57. [CrossRef]

11. Leach, L.; Zhu, Y.; Chaturvedi, S. Development and Validation of a Real-Time PCR Assay for Rapid Detection of Candida auris from Surveillance Samples. J. Clin. Microbiol. 2018, 56. [CrossRef] [PubMed]

12. Ahmad, A.; Spencer, J.E.; Lockhart, S.R.; Singleton, S.; Petway, D.J.; Bagarozzi, D.A.; Herzegh, O.T. A high-throughput and rapid method for accurate identification of emerging multidrug-resistant Candida auris. Mycoses 2019, 62, 513-518. [CrossRef] [PubMed]

13. Yousfi, H.; Ranque, S.; Rolain, J.M.; Bittar, F. In vitro polymyxin activity against clinical multidrug-resistant fungi. Antimicrob. Resist. Infect. Control 2019, 8. [CrossRef] [PubMed]

14. Yousfi, H.; Cassagne, C.; Ranque, S.; Rolain, J.M.; Bittar, F. Repurposing of ribavirin as an adjunct therapy against invasive Candida strains in an in vitro study. Antimicrob. Agents Chemother. 2019, 63. [CrossRef]

15. Lagier, J.C.; Khelaifia, S.; Alou, M.T.; Ndongo, S.; Dione, N.; Hugon, P.; Caputo, A.; Cadoret, F.; Traore, S.I.; Seck, E.H.; et al. Culture of previously uncultured members of the human gut microbiota by culturomics. Nat. Microbiol. 2016, 1, 16203. [CrossRef]

16. Bardet, L.; Le Page, S.; Leangapichart, T.; Rolain, J.M. LBJMR medium: A new polyvalent culture medium for isolating and selecting vancomycin and colistin-resistant bacteria. BMC Microbiol. 2017, 17, 220. [CrossRef]

17. Jung, B.; Hoilat, G.J. MacConkey Medium; n: StatPearls [Internet]; StatPearls Publishing: Treasure Island, FL, USA, 2021.

18. Calvo, B.; Melo, A.S.A.; Perozo-Mena, A.; Hernandez, M.; Francisco, E.C.; Hagen, F.; Meis, J.F.; Colombo, A.L. First report of Candida auris in America: Clinical and microbiological aspects of 18 episodes of candidemia. J. Infect. 2016, 73, 369-374. [CrossRef]

19. Ruiz-Gaitán, A.; Moret, A.M.; Tasias-Pitarch, M.; Aleixandre-López, A.I.; Martínez-Morel, H.; Calabuig, E.; Salavert-Lletí, M.; Ramírez, P.; López-Hontangas, J.L.; Hagen, F.; et al. An outbreak due to Candida auris with prolonged colonisation and candidaemia in a tertiary care European hospital. Mycoses 2018, 61, 498-505. [CrossRef]

20. Chowdhary, A.; Sharma, C.; Duggal, S.; Agarwal, K.; Prakash, A.; Singh, P.K.; Jain, S.; Kathuria, S.; Randhawa, H.S.; Hagen, F.; et al. New clonal strain of Candida auris, Delhi, India. Emerg. Infect. Dis. 2013, 19, 1670-1673. [CrossRef]

21. L'Ollivier, C.; Cassagne, C.; Normand, A.C.; Bouchara, J.P.; Contet-Audonneau, N.; Hendrickx, M.; Fourquet, P.; Coulibaly, O.; Piarroux, R.; Ranque, S. A MALDI-TOF MS procedure for clinical dermatophyte species identification in the routine laboratory. Med. Mycol. 2013, 51, 713-720. [CrossRef]

22. De Almeida, J.N.; Francisco, E.C.; Hagen, F.; Brandão, I.B.; Pereira, F.M.; Dias, P.H.P.; De Miranda Costa, M.M.; De Souza Jordão, R.T.; De Groot, T.; Colombo, A.L.; et al. Emergence of Candida auris in Brazil in a COVID-19 Intensive Care Unit. J. Fungi 2021, 7, 220. [CrossRef]

23. Allaw, F.; Kara Zahreddine, N.; Ibrahim, A.; Tannous, J.; Taleb, H.; Bizri, A.R.; Dbaibo, G.; Kanj, S.S. First Candida auris Outbreak during a COVID-19 Pandemic in a Tertiary-Care Center in Lebanon. Pathogens 2021, 10, 157. [CrossRef] 
24. Chowdhary, A.; Sharma, C.; Meis, J.F. Candida auris: A rapidly emerging cause of hospital-acquired multidrug-resistant fungal infections globally. PLoS Pathog. 2017, 13, e1006290. [CrossRef] [PubMed]

25. De Jong, A.W.; Dieleman, C.; Carbia, M.; Tap, R.M.; Hagen, F. Performance of two novel chromogenic media for the identification of multidrug-resistant candida auris compared with other commercially available formulations. J. Clin. Microbiol. 2021, 59. [CrossRef]

26. Das, S.; Singh, S.; Tawde, Y.; Chakrabarti, A.; Rudramurthy, S.M.; Kaur, H.; Shankarnarayan, S.A.; Ghosh, A. A selective medium for isolation and detection of Candida auris, an emerging pathogen. J. Clin. Microbiol. 2021, 59. [CrossRef] [PubMed]

27. Borman, A.M.; Fraser, M.; Johnson, E.M. CHROMagarTM Candida Plus: A novel chromogenic agar that permits the rapid identification of Candida auris. Med. Mycol. 2021, 59, 253-258. [CrossRef] [PubMed]

28. Nadeem, S.G.; Hakim, S.T.; Kazmi, S.U. Use of CHROMagar Candida for the presumptive identification of Candida species directly from clinical specimens in resource-limited settings. Libyan J. Med. 2010, 5, 1-6. [CrossRef] 